\title{
Théologiques
}

\section{Le moi et Dieu selon Maître Eckhart}

\section{Olivier Boulnois}

Volume 16, numéro 2, 2008

L'individualisation de la relation religieuse

URI : https://id.erudit.org/iderudit/001714ar

DOI : https://doi.org/10.7202/001714ar

Aller au sommaire du numéro

Éditeur(s)

Faculté de théologie et de sciences des religions, Université de Montréal

ISSN

1188-7109 (imprimé)

1492-1413 (numérique)

Découvrir la revue

Citer cet article

Boulnois, O. (2008). Le moi et Dieu selon Maître Eckhart. Théologiques, 16(2), 49-66. https://doi.org/10.7202/001714ar
Résumé de l'article

Le Sermon 52 d'Eckhart se situe au croisement de l'aristotélisme et de l'augustinisme : l'homme accède à la béatitude par la contemplation, il a pour modèle la divinisation du chrétien. Pour Eckhart, seul le détachement permet à l'homme d'y accéder, par un triple renoncement : à la concupiscence de la chair, à la convoitise du savoir et au désir de dominer. Dans ce détachement, devient-il un ça, un intellect abstrait, impersonnel? Cesse-t-il d'être un moi? Apparemment, puisque Dieu seul peut dire " moi », selon Eckhart. Mais en réalité, pas du tout, car le sermon a été prononcé pour la fête de tous les saints, unis à Dieu sur le modèle du Fils avec son Père : une seule essence, mais deux personnes.
Ce document est protégé par la loi sur le droit d'auteur. L'utilisation des services d'Érudit (y compris la reproduction) est assujettie à sa politique d'utilisation que vous pouvez consulter en ligne.

https://apropos.erudit.org/fr/usagers/politique-dutilisation/ 


\title{
Le moi et Dieu selon Maître Eckhart
}

\author{
Olivier BoulnOIs" \\ Sciences religieuses \\ École Pratique des Hautes Études
}

Vue de l'extérieur, l'œuvre de Maître Eckhart s'inscrit sans doute dans le cadre d'une évolution des pratiques religieuses et d'une naissance de l'individu à la fin du Moyen Âge. Mais pour analyser le contenu de son œuvre, il n'est pas certain que ces concepts soient pertinents. En effet, au Moyen Âge, le concept d' «individu », plutôt qu'au problème du sens de l'existence humaine, renvoie à une question métaphysique classique, celle de l'individuation des êtres : à l'intérieur de l'espèce humaine, qu'est-ce qui distingue Pierre de Paul, X de Y ? Et le monde latin ne connaît pas de mot précis pour désigner ce que nous appelons maintenant une « religion » : chez saint Thomas, par exemple, la religion est une vertu, qui constitue un sousensemble de la vertu de justice; dans la mesure où nos actes de piété consistent à rendre à Dieu ce qui est à Dieu, la religion est une province de l'art de rendre à chacun ce qui lui revient. Je préfère donc traiter, plus exactement, de la relation entre moi et Dieu.

Avec cette question, on ne perd pas tout à fait de vue l'horizon de l'individu et de l'individuation, du moins quand on s'interroge sur le moi. « Je suis devenu pour moi-même une question ", dit Augustin dans les Confessions. Et nous savons que cette question devient aiguë, précisément au moment où se pose la question de sa conversion, c'est-à-dire de son retour à Dieu.

Qu'est-ce que le moi? Quelque chose de personnel: ce que je suis? D'unique: le moi ? D'identique : le même? Mais le moi est-il connaissable comme un objet? N'est-il pas plutôt le support inconnaissable de tous les

* Olivier Boulnois est directeur d'études à l'École Pratique des Hautes Études (Religions et philosophies dans le christianisme au Moyen Âge). Ses travaux portent sur la philosophie médiévale, l'histoire de la théorie des images et la philosophie de la liberté. Il a publié, en 2008 Au-delà de l'image. Une archéologie du visuel au Moyen Âge ( $V^{e}-X V I^{e}$ siècle), Paris, Seuil (Des Travaux). 
accidents qui sont mes propriétés? Quand je pose la question «qui suisje ? ", j'achoppe sans cesse sur des réalités préconstituées, dans lesquelles je ne me reconnais pas nécessairement: je suis le résultat d'une série de causalités, je suis né avec tels caractères héréditaires, j'ai grandi dans un lieu que je n'ai pas choisi, j'ai reçu une culture qui s'est imposée à moi, je suis pris dans une histoire que je n'ai pas faite. Notre être nous a été donné avant que nous puissions choisir quoi que ce soit. Où situer alors ce qui est vraiment moi, ce que je ressens comme inviolable et irréductible à toutes ces données? Je peux m'affirmer comme un individu, une unicité, totalement différente de tous les autres, et nier le caractère unique et irréductible de tous les autres «moi ». Mais pourquoi les autres ne seraient-ils pas comme moi ? Mes prétentions à l'autosuffisance ne sont-elles pas dérisoires? La passion avec laquelle nous affirmons notre unicité et notre inviolabilité a-t-elle un fondement ? Pourquoi me cramponner à ce que j'ai en propre, à mes propriétés ou mes qualités, alors que la seule chose qui distingue le moi de tous les autres, ce sont justement ces propriétés dont je ne suis pas l'auteur? Pourquoi revendiquer notre moi comme la source et l'origine du sens, alors qu'il est seulement l'entrecroisement de toutes nos servitudes?

Et qu'est-ce que Dieu? Quelque chose d'unique? D'identique? De personnel ? Et Dieu est-il connaissable comme un objet? N'est-il pas plutôt le support inconnaissable de tous les attributs que nous extrapolons à partir de nos expériences?

Et enfin, qu'est-ce qui est le plus incompréhensible? Dieu ou l'homme? Ou bien ne faut-il pas établir un lien entre ces deux abîmes d'incompréhensibilité ? "Que je me connaisse et que je te connaisse», disait encore Augustin. Est-ce en reconnaissant l'incompréhensibilité du moi qu'on pourra concevoir l'incompréhensibilité de l'absolu ? Ou bien, à l'inverse, en saisissant l'inconnaissable hors de nous que l'on pourra contempler son image en nous?

Pour saisir ce qui nous constitue en un moi, il faut donc tout repenser. Et commencer par nous désapproprier de tout ce que nous avons en propre. C'est pourquoi j'ai centré mon étude sur Eckhart, le théoricien de la désappropriation. Pour des raisons d'économie, je m'en suis tenu essentiellement à la lecture des deux premiers tiers du Sermon 52.

\section{La question de la béatitude}

Il faut d'abord replacer notre sermon dans une perspective plus vaste, celle du problème de la béatitude, depuis ses deux sources, grecque et biblique. 
Du côté de la pensée grecque, la grande source est Aristote. Pour Aristote, tous les hommes désirent être heureux, c'est la première proposition de l'Éthique à Nicomaque. Mais si l'homme tâtonne grâce à la prudence et dans l'action, pour atteindre autant que possible au bonheur, il est acquis pour Aristote que la plus haute forme de félicité consiste dans la vie spéculative (ou contemplative), laquelle culmine à son tour dans la considération des réalités les plus hautes, les réalités divines.

Mais qu'est-ce qui peut arriver à cette contemplation? - L'intellect. Selon Aristote, celui-ci est d'abord intellect possible, pure puissance, absolument indéterminée. Il faut que l'intellect ne soit rien pour qu'il puisse devenir tout ce qu'il pense. Il en résulte que l'intellect n'est rien en luimême, rien avant de penser: "Cela donc qu'on appelle intelligence de l'âme [...] n'est en acte aucun des êtres avant de penser » (De l'Âme, III, 4, 429 a 17-23). Comme le dira à son tour Eckhart: "L'intellect, en tant qu'intellect, n'est rien de ce qu'il pense, mais il faut qu'il soit "non-mélangé”, "n’ayant rien de commun avec autre chose", afin de les penser toutes, comme il est dit dans le Traité de l'âme III, de même que la vue doit n'avoir aucune couleur pour les voir toutes. Si donc l'intellect, en tant qu'intellect, n'est rien, l'acte de penser, lui non plus, n'est pas un être ${ }^{1}$ ».

Mais l'intellect qui pense le plus haut intelligible, est-ce nous? L'analyse d'Aristote introduit de nombreuses difficultés. D'une part, puisque l'intellect n'a d'être que lorsqu'il est en acte de penser, il en résulte qu'il doit d'abord être en acte de penser autre chose pour devenir à son tour une réalité connaissable. Il ne peut se connaître et se penser lui-même que par un acte second: la pensée est d'abord pensée d'un autre, elle ne peut revenir sur elle-même que par une réflexion seconde. Par conséquent, ce qui est connu n'est pas le moi, ou le sujet de la pensée, mais toujours une pensée ou une connaissance. Je ne me connais jamais qu'indirectement, à partir de mes connaissances d'objet. L'âme ne se connaît donc qu'indirectement, elle se rapporte à soi-même comme à un autre.

Il reste que l'homme peut, à force de penser, devenir pensant. Il deviendra intelligible pour lui-même, précisément dans la mesure où il deviendra intelligent, c'est-à-dire pensant. La définition de l'homme en tant qu'homme réside alors dans l'intellect, c'est-à-dire dans sa capacité à devenir toutes choses par la pensée. Comme le dit encore Eckhart:

1. Eckhart, Quaestiones parisienses q.1, \$.2, trad. fr. Kaluza et al., 169, modifiée. 
Voilà ce qu'Aristote attribue à l'homme pour dire ce qui en fait un homme: il comprend toutes les images et toutes les formes [...]. Or je veux moi aussi montrer ce qu'est un homme. "Homo ", cela veut dire un homme à qui est impartie une substance qui lui donne être et vie et en fait un être doué d'intellect. Un homme doué d'intellect est quelqu'un qui se comprend lui-même intellectuellement et qui est en lui-même détaché de toutes matières et de toutes formes ${ }^{2}$.

Mais la béatitude est un attribut divin. L'homme peut-il y avoir part? La pensée d'Aristote est écartelée selon une tension tragique: la divinisation est la fin nécessaire de l'existence humaine, elle est le comble auquel tout homme aspire en désirant le bonheur. Mais Aristote se rappelle la leçon de la tragédie classique, qui expose la triste destinée de l'homme, lorsqu'il essaie d'égaler les dieux. Aristote reconnaît, certes, que la recherche du bonheur pousse l'homme à transgresser la limite entre le divin et l'humain. Et il rejette la position de ceux qui conseillent à l'homme de «borner sa pensée aux choses humaines, et mortel, aux choses mortelles ${ }^{3}$ ». L'homme doit "dans la mesure du possible, s'immortaliser»(athanatizein) ${ }^{4}$ ». Mais dans quelle mesure est-ce possible? "Une vie de ce genre sera trop élevée pour la condition humaine: car ce n'est pas en tant qu'homme que l'on vivra de cette façon, mais en tant que quelque élément divin est présent en nous » (1177 b 27). La félicité est un attribut divin; elle n'est accessible à l'homme que dans la mesure où il est intellect, car «l'intellect est quelque chose de divin par comparaison avec l'homme» (b 31). Poursuivre la félicité est nécessaire pour l'homme, mais la posséder durablement, sur le mode divin, est impossible pour lui. Le désir d'éternité est donc pour l'homme un effort perpétuel et contradictoire, une tension vers l'inatteignable.

Si ce n'est pas l'homme, qu'est-ce qui l'atteindra? "Chez les dieux, la vie est tout entière bienheureuse »; pourtant, les hommes n'atteignent le bonheur "que dans la mesure où une certaine ressemblance avec l'activité divine se trouve en $\mathrm{eux}^{5}$ » $:$ l'acte de penser est divin et la vie de l'homme peut ressembler à celle des dieux quand il médite. Mais qu'est-ce qui médite? L'intellect et non le composé âme-corps. C'est pourquoi «le bonheur de l'intellect est séparé6 ${ }^{6}$ : il est séparé de nous, composés d'un corps et d'une

2. Eckhart, Sermon allemand 15, «Homo quidam nobilis» DW I, 249; trad. Libera 1993, 314, légèrement modifiée.

3. Aristote, Éthique à Nicomaque x, 7, 1177 b 32-33.

4. Éthique à Nicomaque $\mathrm{x}, 7,1177$ b 33.

5. Éthique à Nicomaque $\mathrm{x}, 9,1178 \mathrm{~b} 25 \mathrm{~s}$.

6. Éthique à Nicomaque $\mathrm{x}, 8,1178$ a 22. 
âme, et il ne devient accessible que dans la mesure où nous devenons intellect en acte. À moins que l'on n'identifie l'homme à ce qu'il y a de plus élevé en lui : l'intellect. Mais dans ce cas, ce n'est encore qu'un aspect de sa nature, le sommet de l'âme, et non celle-ci tout entière. Par conséquent, nous n'atteignons la félicité que partiellement et par intermittence.

Or, la tradition biblique, dans son versant chrétien, propose une autre approche de la béatitude. D'une part, les béatitudes promettent d'une manière paradoxale l'accès au Royaume de Dieu à ceux qui en semblent le plus exclus. De l'autre, le Christ reprend un passage des Psaumes "vous êtes des dieux, et les fils du Très-Haut » (Psaume 81,6), et l'applique à ses disciples (Jean 10,35). Il y a là un retournement complet du sens de l'existence humaine, comme l'a remarqué avec force M. Balmary (2002, 393-414). Elle y voit une véritable brèche dans le mur de l'humanité. La divinisation cesse d'être un désir contradictoire et coupable, elle devient une possibilité offerte au croyant s'il se conforme à la parole de Dieu. Depuis le concile d'Éphèse (431), qui affirmait la parfaite union des deux natures, humaine et divine, dans la personne du Christ, la divinisation du chrétien est considérée comme possible, puisqu'elle a son modèle parfait dans le Christ.

Dès l'œuvre de saint Augustin, la divinisation du chrétien apparaît comme la solution au problème du désir du bonheur, dont il rappelle la puissance dès le début du traité de La Vie heureuse. Car la poursuite du bonheur cesse d'être une tension contradictoire. Elle peut être accomplie, et elle l'a été par son modèle, le Christ. À son tour, Augustin déclare: "chacun est tel qu'est son amour; tu aimes la terre? tu seras terre. Tu aimes Dieu, que dirais-je ? tu seras Dieu. Je n'ose pas le dire de moi-même, mais écoutons les Écritures: "vous êtes des dieux, et les fils du Très-Haut" (Psaume 81,6 ${ }^{7}$ ». Ce qu'il n'ose pas dire de lui-même, Augustin le trouve dans l'Écriture : l'homme peut accéder à la divinisation. Il y a là une mutation profonde dans l'anthropologie, une nouvelle compréhension du bonheur humain, et des rapports entre l'humain et le divin. Cette théorie s'accompagne à partir d'Augustin d'une identification permanente du moi avec la certitude d'être celui qui pense ${ }^{8}$. C'est donc bien moi, moi qui pense, et moi qui suis pourtant en chair et en os, qui accède à la béatitude, sans être dépouillé de rien de ce qui fait mon identité et ma singularité.

7. Augustin, Sur la Première Épître de saint Jean 2,14 (PL 35, 1997).

8. C’est ce qu'on a appelé le «cogito augustinien»; voir Boulnois $(1999,151-222)$ et Bermon (2001). 


\section{Le dépouillement du moi}

Or, Eckhart s'inscrit précisément au croisement de ces deux traditions, augustinienne et aristotélicienne.

Si l'être que je suis peut se conquérir comme un moi, il lui faut changer d'orientation, se détourner du monde, mais aussi de lui-même, en tant qu'objet mondain, et revenir à soi comme à un moi véritable. Comment estce possible? Cette aspiration qui est en chacun de nous n'est pas une loi de la nature qui s'accomplirait nécessairement : il appartient à chacun de nous d'y parvenir ou d'y échouer. En effet, tant que nous sentons et pensons, nous pouvons nous tourner vers les images, vers nos perceptions du monde sensible, ou bien en revenir à l'essentiel en nous, à ce qui n'a pas d'image. Il faut se libérer des images pour atteindre son essence véritable. Le détachement est ainsi l'acte de se vider de toutes les images ou de toutes les formes (Bild), de se désimager (entbilden), pour se former (bilden) et se réformer (überbilden). La véritable éducation (Bildung) doit aller non vers l'accumulation de connaissances et d'images, mais vers la simplification, vers le dépouillement des formes (Entbildung). Il faut parvenir ainsi au dépassement de toute image - entbliden, ymagine denudari, "être dépouillé par l'image » invisible, c'est en même temps être «dépourvu d'image » visible (Wackernagel, 1991). Aucune réforme n'est possible sans cette reformation de l'intellect. La formation est ainsi la suppression de toutes les images, elle débouche sur l'acte de se trouver comme moi sans image: "On demande où l'être de l'image se trouve le plus proprement: dans le miroir ou dans ce dont elle provient ? Elle est le plus proprement dans ce dont elle provient. L'image est en moi, elle vient de moi, elle vient à moi. Tant que le miroir se trouve en face de mon visage, mon image y est aussi; mais que le miroir tombe, et l'image disparaîtra'. " Comme l'ange, miroir de Dieu, l'image est suspendue à l'original. L'être propre de l'image n'est pas dans le sujet qui l'accidente, mais dans l'origine. Or, puisque nous ne connaissons que par des images et des formes abstraites de l'expérience, cela signifie que nous devons nous atteindre nous-mêmes comme inconnaissables.

Le Sermon 52 porte sur la béatitude comme fin de l'existence. Il commente la phrase de Matthieu 5,3: «Bienheureux les pauvres en esprit, car le royaume des cieux est à eux ", c'est-à-dire précisément la première des béatitudes dans la liste de l'Évangile. Avec un humour pré-claudélien, Eckhart ajoute « je vous prie [...] de bien vouloir comprendre cette vérité

9. Sermon allemand $\mathrm{n}^{\circ}$ 9: «Quasi stella matutina " (DW I, 153-154); trad. Libera (1993, 279) modifiée. 
si vous le pouvez. Mais si vous ne la comprenez pas, ne vous inquiétez pas. " Avant de déployer le sens de cet énoncé, Eckhart rappelle que son interprétation ne va pas de soi : il faut que l'auditeur grandisse avec le texte pour le comprendre, et tous n'en sont pas capables. C'est donc un énoncé au statut herméneutique particulier, qui sélectionne son destinataire et l'élève vers la béatitude, mais que ne pénètrent pas ceux qui en sont incapables.

Eckhart donne à ce verset un triple commentaire: "Celui-là est un homme pauvre qui ne veut rien, ne sait rien et n'a rien ${ }^{10}$.» Trois éléments, donc, inséparables dans la réalité, mais qu'Eckhart examine successivement: ne rien vouloir, ne rien savoir, ne rien avoir. Pourquoi cette triple désappropriation?

Je pense que ces trois mouvements sont en réalité la négation des trois libidos de saint Augustin, élaborées dans son commentaire du verset de la première Épitre selon saint Jean «la concupiscence de la chair, la concupiscence des yeux et l'orgueil de la vie ${ }^{11} \gg$ : ne rien désirer pour soi-même s'oppose à la libido sententiendi; ne rien savoir, évidemment, à la libido sciendi; ne rien posséder, à la libido dominandi.

1) Pourquoi ne rien vouloir? "Ce sens est mal compris par certaines gens, par ceux qui, en faisant pénitence et en se livrant aux exercices extérieurs, ce qu'ils estiment fort, restent attachés à leur être propre » (349). On les appelle saints à cause de leur apparence extérieure, mais en eux-mêmes ce sont des ânes. En effet «ils entendent par là que l'homme doit vivre de façon à ne plus faire sa propre volonté en quoi que ce soit, mais à s'efforcer de faire la volonté très chère de Dieu» (349). Or, la pauvreté ne consiste pas à faire un effort, ni à faire la volonté de Dieu. Car cela, c'est encore vouloir faire la volonté de quelqu'un: "cet homme a encore une volonté, par laquelle il veut satisfaire la volonté de Dieu, et ce n'est point là la vraie pauvreté » (350). "Car celui-là seul est un homme pauvre qui ne veut rien et ne désire rien» (350). Pour posséder vraiment la volonté, il faut que l'homme se vide de sa volonté créée, ainsi qu'il le faisait au moment où il n'était pas encore. Il nous faut donc anéantir la volonté - et pas seulement notre volonté propre - , parce qu'il nous faut revenir au néant qui a précédé la création. Mais même s'il faut dépasser tout l'être, surpasser toute ontologie et toute méta-

10. Ce sermon est un des derniers prononcés par Eckhart; trad. Libera (1993, 349).

11. I Jean 2,16 . 
physique, nous retrouvons alors une essence. Car nous retrouvons ce que nous étions dans la pensée divine avant d'être créés. "Quand j'étais encore dans ma cause première [...] Je ne voulais rien, je ne désirais rien, car j'étais un être vide (ledic), me connaissant moimême dans la jouissance de la vérité12. » Dans la pensée divine, j'étais libre, c'est-à-dire vide de toutes choses, et Dieu n'était pas extérieur à moi. D'où la proposition hyperbolique: "Là j'étais libre de Dieu » (350). Par conséquent, être pauvre en volonté, c'est ne rien vouloir, et ne rien vouloir, c'est vouloir aussi peu que l'homme, au temps où il n'était pas encore. C'est non seulement vouloir être vide du monde extérieur, vide de toute volonté, mais paradoxalement aussi, vouloir être vide de Dieu. Si Dieu est sans forme et vide d'images, le lieu de l'âme en Dieu est sans forme, il est vide. Autrement dit, comme le dit le Sermon 83, dans ce retour à l'unité avec Dieu, "l'être nu de l'âme reposant passivement en lui-même rencontre l'être nu, sans forme, de l'unité divine, qui est l'être suressentiel ${ }^{13}{ }^{\prime}$. Il faut que l'homme se libère de toute forme pour atteindre un Dieu libre de toute forme.

2) Pourquoi ne rien savoir? Eckhart propose une série d'approfondissements successifs :

Nous avons dit parfois que l'homme devrait vivre comme s'il ne vivait ni pour lui-même, ni pour la vérité, ni pour Dieu. Mais maintenant nous parlons autrement et nous irons plus loin en disant: Pour arriver à cette pauvreté, l'homme doit vivre de telle manière qu'il ne sache pas même qu'il ne vit ni pour lui-même, ni pour la Vérité, ni pour Dieu. Bien plus: Il faut qu'il soit à tel point vide de tout son propre savoir qu'il ne sache, ni ne connaisse, ni ne sente que Dieu vit en lui. Plus encore: Il faut qu'il soit vide de toute connaissance qui pourrait vivre en lui (p. 351).

Selon le même principe, Eckhart montre que l'homme doit rester aussi vide de son propre savoir qu'il le faisait au temps où il n'était pas encore.

3) Pourquoi ne rien avoir? Cette évacuation de toutes choses peut sembler dégager une place où l'homme pourrait recevoir Dieu ou l'action de Dieu. C'est ce que les premiers sermons d'Eckhart semblent établir: "J'ai souvent dit, et de grands maîtres le disent également, qu'il faut que l'homme soit vide de toutes choses et de toutes œuvres, intérieures aussi bien qu'extérieures, au point de pouvoir être un

12. Je modifie légèrement la traduction d'A. de Libera (1993, 350).

13. Trad. J. Ancelet-Hustache $(1979,151)$ modifiée par Libera (1993, 487). 
lieu propre pour Dieu, où Dieu puisse œuvrer» (353). Mais il faut aller plus loin: "Ce que Dieu recherche dans ses opérations, ce n'est point que l'homme ait en lui un lieu où Dieu puisse opérer; car il n'y a vraiment pauvreté en esprit que lorsque l'homme est à tel point libéré de Dieu et de toutes ses œuvres que Dieu, s’il voulait opérer dans l'âme, devrait être lui-même le lieu de son opération. Or cela, Dieu le fait volontiers» (353). En effet, Dieu est acte pur, acte pur de se penser, de se connaître, et acte pur de se donner à tout ce qui est divin. Il se répand ainsi dans tout ce qui n'est pas créature mais pure intellectualité, être essentiel. Or, si l'intellect parvient à se dépouiller de tout ce qui est créé, Dieu ne peut que se donner à lui, à son être essentiel. En agissant dans l'âme, Dieu agit en lui-même, «il est le lieu propre de son opération » (353). Par conséquent, c'est en se détachant de toutes choses, y compris de Dieu, que l'homme s'unit à Dieu. Car Dieu n'est pas extérieur à l'homme, seules les réalités créées sont extérieures les unes aux autres, et en enlevant toutes les créatures, l'homme retrouve Dieu en lui, ayant dépassé ce qui est lui-même, il retrouve ce qui est « plus intime à lui-même que luimême ", comme disait saint Augustin.

Qu'est-ce que le moi lorsqu'il est devenu Dieu ? Le moi peut-il ne rien vouloir, alors que l'homme désire toujours quelque chose, ce qui lui apparaît comme bon? (Aristote, Éthique à Nicomaque I, 1). Le moi peut-il ne rien savoir, alors que l'essentiel de l'homme est la pensée? (Éthique à Nicomaque $\mathrm{x}$ ). Le moi peut-il ne rien avoir, alors même qu'il lui faut agir et être formé par ses propres actions, ce qui est la définition de l'habitus (disposition acquise par l'habitude, Éthique à Nicomaque vi). En réalité, ce vouloir, ce savoir et cet avoir sont toujours le vouloir, le savoir et l'avoir d'un objet, d'un autre ou d'un être. C'est le moi qui ne doit rien vouloir, savoir ni avoir d'extérieur, mais se tourner vers lui-même, sans altérité, sans être: c'est dans le néant d'objet qu'il accède à soi.

Eckhart évoque en chemin un débat entre la tradition franciscaine et la tradition dominicaine: "sur quoi se fonde avant tout la Béatitude? Quelques maîtres [surtout franciscains] ont dit qu'elle est fondée sur l'amour, d'autres [surtout dominicains], qu'elle repose sur la connaissance et l'amour; ces derniers parlent déjà mieux» (351). Mais Eckhart propose de remonter en deçà de cette opposition: «il y a quelque chose dans l'âme d'où émanent la connaissance et l'amour. Cela ne connaît pas et n'aime pas; ce sont les puissances de l'âme qui connaissent et aiment» (351). 
En effet, comme le dit le Sermon 28: «il y a quelque chose dans l'âme qui dépasse l'essence créée de l'âme, quelque chose que rien de créé ne touche, quelque chose qui n'est rien » (325). Cela est apparenté à la nature divine, et cela transcende toutes choses: "Si tu pouvais t'anéantir toi-même, ne fût-ce qu'un instant ou même moins qu'un instant, alors tout ce que cela est en soi-même t'appartiendrait en propre» (325). Chaque homme possède en soi un quelque chose dans l'âme qui n'est pas l'âme elle-même, mais son principe, son origine, c'est vers ce quelque chose que l'intellect possible doit se tourner, pour devenir cela. Et cela, c'est le moi le plus propre qui puisse m'appartenir.

Il existe en l'âme un cela, ou un çà, plus fondamental que les puissances dont nous faisons l'expérience: "C'est pourquoi cela ne peut pas non plus savoir que c'est Dieu qui agit en lui; il est lui-même le même qui jouit de lui-même, comme le fait Dieu » (351). Le pauvre en esprit est celui qui ne sait rien d'aucune chose, ni de Dieu, ni de la créature, ni de lui-même. C'est dans cette auto-affection du ça, sans rapport théorique à soi, au monde et à Dieu, qu'il jouit de lui-même et qu'il atteint la béatitude. L'aspect le plus anonyme de l'âme, le ça, se trouve investi exactement des propriétés de la science par laquelle Dieu se connaît lui-même selon la Métaphysique L d'Aristote. En effet, le çà en nous est ce qui pense, un intellect pur, détaché de toutes choses, transcendant l'être, et qui coïncide avec la pensée divine. En termes scolastiques, ce "quelque chose dans l'âme » est incréé et incréable - c'est Dieu présent au plus intime de moi-même. Attention : ce «quelque chose » de divin est "dans l'âme ", il n'est pas "de l'âme ", ce que n'ont pas compris ceux qui l'accusèrent de panthéisme, à commencer par la Commission d'enquête (inquisitio) qui a examiné son œuvre. Eckhart appelle également cela une «étincelle dans l'âme », " un fond incréé». Dans la pensée, le fond de l'âme rejoint le fond de la divinité.

Eckhart se situe en effet à la suite d'une lignée qui commence avec Aristote, nous l'avons vu, qui se poursuit avec Averroès, pour lequel l'âme ne pense que dans la mesure où, dans l'acte de connaître, elle s'unit à un intellect transcendant, et qui s'achève avec Dietrich de Freiberg, parlant d'un intellect transcendant dans l'âme. Mais c'est Eckhart qui lui adjoint le qualificatif d'incréée, la rapprochant ainsi de la pensée d'Augustin. Le moi en tant que moi n'est donc pas l'âme, mais quelque chose de divin, l'intellect incréé et incréable. C'est seulement ainsi que le moi peut être lui-même, c'est-à-dire être un soi, unique, unifié et permanent. Une fois qu'il sera devenu un tel soi, il sera capable de connaissance de soi, d'amour de soi et 
de possession de soi. On remarquera que ces trois propriétés sont finalement rendues par Eckhart à l'intellect détaché de toutes choses, au terme de chaque analyse.

\section{L'union à Dieu}

Qui peut dire "moi » ? En réalité, seul Dieu peut le faire, lui seul est une identité permanente, unique et unifiée. Comme le dit le Sermon 28: "Le mot latin ego, qui signifie “je”, n'appartient à personne, il n'est propre qu'à Dieu seul dans son unité » (326). Nous ne sommes pas vraiment des «moi », sauf dans la mesure où nous sommes unis à Dieu.

Mais quelle différence y a-t-il entre l'union de l'homme à Dieu et l'union de Dieu à l'homme? - Aucune. Il faut comprendre que ce processus de détachement de l'homme n'est que le revers d'un mouvement de manifestation de Dieu. La désappropriation de l'homme et l'éthique de la pauvreté sont rendues possibles par la pauvreté divine, par un mouvement symétrique de désappropriation en Dieu. Nous l'avons dit, en se dépouillant de toutes les images sensibles, l'intellect en l'homme devient divin. Il devient exactement une image de Dieu égale à l'original. Se dépouiller des images, c'est devenir le pur miroir de l'absolu divin, de même nature que lui. En effet, avant d'être une représentation, un substitut de l'objet, l'image est la présentation de l'objet, elle est constitutive de la forme. L'image (Bild) est une formation, et non une copie (Abbild). Elle rend visible l'original. Ainsi, il existe pour l'essence divine un équivalent du stade du miroir chez Lacan: l'image ne reproduit pas une vision préalable que l'on pourrait avoir de l'objet, elle la constitue. En ce sens, l'image est toujours l'image de l'invisible; elle rend visible ce qui sans elle ne peut pas être vu. Appliquée à la relation entre l'image de Dieu et Dieu, cette analyse signifie que Dieu ne se manifeste que dans une image adéquate à l'original. Sans l'image parfaite où Dieu se manifeste, et à laquelle l'intellect dans l'homme peut accéder, Dieu serait un fond sans forme. Mais il ne se manifeste que dans la cime de l'âme, et dans le même mouvement, il constitue l'âme humaine dans son essence comme image de lui-même, comme image de l'original divin ${ }^{14}$.

Et qui est cette image? Pour Eckhart, devenir cette image, c'est devenir le Fils de Dieu, image de Dieu le Père. On pourrait voir dans cette analyse une glose d'une célèbre phrase de saint Irénée: "Le Fils est le visible du Père, et le Père est l'invisible du Fils. » Devenir intellect pur, ou image de

14. Pour toute cette analyse, voir Boulnois (2008, 289-332). 
Dieu, c'est en même temps participer à l'acte par lequel l'invisibilité divine se manifeste dans le Fils.

Beaucoup de maîtres prétendent que l'image est issue de la volonté et de la connaissance. Il n'en est pas ainsi. Je dis bien plutôt que cette image est une expression d'elle-même sans volonté ni connaissance. Je vais vous en donner une comparaison. On place un miroir devant moi; que je le veuille ou non, sans ma volonté et ma connaissance, je me reflète dans le miroir. Cette image n'est pas celle du miroir, elle n'est pas non plus celle d'elle-même, l'image provient bien plutôt de celui dont elle a reçu son essence et sa nature. Quand le miroir qui était devant moi est enlevé, je ne me reflète pas plus longtemps dans le miroir, car je suis cette image elle-même.

Encore une autre comparaison. Quand une branche émane de l'arbre, elle porte le nom et l'être de l'arbre, ce qui sort est identique à ce qui demeure à l'intérieur, et ce qui reste à l'intérieur est identique à ce qui sort. Ainsi la branche est une expression d'elle-même.

Je dis absolument de même pour l'image de l'âme. Ce qui sort est identique à ce qui reste à l'intérieur et ce qui reste à l'intérieur est identique à ce qui sort. Cette image, le Fils l'est du Père, et cette image, je la suis, moi (illa sum ego ymago $)^{15}$.

Je suis l'image de Dieu en Dieu, je m'identifie ainsi au Fils. En devenant moi, je participe au mouvement d'engendrement du Fils. Pourquoi le contemplateur s'identifie-t-il à un fils ? Parce qu'il est engendré dans l'acte de connaître son principe. Le contemplateur s'identifie à un «fils", parce que son unité avec le Père advient dans l'acte même par lequel il connaît son principe. Par conséquent, la connaissance de soi est ce en quoi advient la connaissance du principe : connaître le principe, pour le moi, c'est rejoindre l'acte même par lequel est engendrée l'unité avec le principe qui lui donne l'être. Et réciproquement, seul celui qui est engendré par le principe peut connaître le principe, tant sa transcendance est grande : «Le supérieur n'est connu que par lui seul et par ce qui est engendré par lui, [par] ce qui ne lui est pas étranger ni n'est autre chose (aliud) que lui qui [l']engendre ${ }^{16}$.»

L'union à Dieu est-elle une confusion? Ce qu'on appelle la mystique a souvent été caractérisé comme une fusion dans l'unité, où l'homme perd son

15. Eckhart, Sermon 16a, "Quasi vas auri solidum", (DW I, 258-259; trad. AnceletHustache, I, 144-145, modifiée); voir Köbele (1998).

16. In Iohannem n. 195 (sur Jn 1,18), LW III, 164 ; trad. fr. (1989, 355-357) légèrement modifiée. Sur cette question, voir Casteigt (2006). 
individualité et se perd dans l' «océan infini de l'essence divine» (selon une formule de Jean Damascène). Est-ce à cela que nous invite Eckhart? Précisément pas. Car Eckhart s'appuie sur le concept d'image. Or l'image fait un avec l'original (elle permet de connaître par son intermédiaire), mais elle reste distincte par le sujet, sans quoi elle n'aurait pas deux termes à unir. En se détachant de toutes choses et en se tournant vers Dieu, l'homme pourra donc dire, comme le Fils, "moi et le Père nous sommes un $(\text { unum })^{17}$ ».

C'est donc le modèle même de l'unité divine qui sert à penser l'union mystique. Rappelons que la théologie trinitaire chrétienne, tout en insistant sur l'unicité et l'unité absolue de Dieu, admet une pluralité de sujets personnels en Dieu. Il n'y a qu'un seul Dieu, Père et Fils, et pourtant le Fils n'est pas le Père: ils ne sont pas un seul (unus). Dieu est une seule essence (unum, neutre), mais il n'est pas une seule personne (unus). De même, l'homme et Dieu, dans leur union, forment une même réalité (unum) mais pas une seule personne $(\text { unus })^{18}$.

Quelle est alors l'origine de mon identité ? Selon Eckhart, si l'identité du moi devient l'identité divine, c'est parce que d'abord la Déité se répand et devient le moi : "Si je dois le connaitre sans médiation, je dois absolument devenir lui, et lui, devenir moi. Je dis plus : Dieu doit absolument devenir

17. Jean 10,30, cité dans Eckhart, In Iohannem, $\mathbb{1 9 3}$ (LW III, 162; trad. fr. 1989, 350); cette distinction vient de S. Bernard, Sermons sur le Cantique, 71, 8: "satis tibi per "unum" et "unus" ipsarum quoque innuitur differentia unitatum, quoniam quidem nec Patri et Filio "unus", nec homini et Deo "unum" poterit convenire». Elle provient des premiers débats sur l'orthodoxie christologique et trinitaire; voir par exemple Grégoire de Nazianze, Lettre théologique 101, I, 21: "Car les deux sont "une seule chose" (hen) par leur union: Dieu d'une part s'est fait homme, l'homme d'autre part a été fait Dieu - ou bien quelle que soit la manière de nommer cela. Je dis ici "une chose" et "une autre" (allo kai allo), à l'opposé de ce qui a lieu pour la Trinité : là, en effet, il y a "un" et "un autre" (allos kai allos) pour que nous ne confondions pas les hypostases, mais non pas "une chose" et "une autre" (allo kai allo) car les trois ne sont qu'une seule chose et la même (bèn kai tauton) par la divinité " (trad. p. 45-47).

18. Eckhart identifie en effet l'union de l'homme à Dieu avec l'union intentionnelle de l'image à l'original qu'elle représente. Or, avec l'original, l'image fait « une seule chose » (unum) et non « un seul [sujet]» (unus) (In Iohannem, $\$ 194)$. Le modèle est bien l'unité du Père et du Fils et non celle de l'homme et de Dieu dans le Christ: «Et c'est ce que dit le Fils, image du Père, en l'annonçant et en le manifestant: "moi et le Père nous sommes un (unum)" ", en raison de l'identité de nature ( $\$ 194$; trad. fr., 352). La pensée d'Eckhart présente bien une mystique de l'essence, parce qu'elle prend pour modèle l'unité trinitaire et non l'unité christologique. C'est précisément aussi pour cela qu'elle n'est pas une mystique de la fusion: la dualité des personnes (des suppôts, chacun restant «unus») demeure. 
moi, et moi absolument Dieu, donc complètement un, si bien que ce lui et ce moi deviennent et sont un "est / ceci" (ist), et dans cette "estance / heccéité" (istigkeit) opèrent éternellement une œuvre, car ce "lui" et ce "moi", c'est-à-dire Dieu et l'âme, sont très féconds ${ }^{19}$. " L'absolu est sans image ni médiation: le connaître en lui-même revient donc à se détacher de toute image et de toute médiation. Dans l'union avec Dieu, l'âme trouve son identification.

Or, Dieu lui-même n'advient à une forme, à une image absolue, que dans la figure du Christ. Le Christ est donc la première désappropriation de la Déité, qui se dépouille de son invisibilité pour se donner dans une forme connaissable.

Il est un moi qui n'est extérieur à aucun autre, et qui n'exclut aucun autre. Toute particularité, toute propriété est consumée par la divinité, unie dans le Christ à l'humanité. Autrement dit, la divinité s'est unie à l'humanité, à tout homme, et non à un homme à l'exclusion des autres. En JésusChrist, l'humanité est unie à Dieu comme à son vrai moi. Elle est alors dépouillée de son moi comme origine de toute appropriation, et dans sa désappropriation, elle s'unit à un autre moi, un moi universel, inépuisable. Au lieu d'être cramponnée à un moi désirant, connaissant ou possessif (les trois rapports à soi dans le Sermon 52), elle est transformée en un moi pauvre, dépossédé, désapproprié, totalement ouvert à l'Autre et à Dieu. Totalement dépouillée, l'humanité est investie par la divinité qui l'occupe tout entière et constitue son moi véritable. Dieu devient le vrai moi de l'homme.

\section{Retrouver le moi}

Qu'est-ce alors que le soi que l'on atteint dans la connaissance de soi ? Le moi doit se dépouiller de tout autre, il doit aussi se dépouiller de lui-même comme d'un autre (objet de connaissance ou d'amour), il doit se dépouiller de l'extériorité de Dieu. Mais il se retrouve comme moi. Eckhart peut en effet reprendre la phrase de saint Paul: «Tout ce que je suis, je le suis par la grâce de Dieu » (I Corinthiens 10,15). Il faut perdre le moi dans sa nature pour le retrouver dans la grâce: "Ce que la grâce a opéré en lui, c'est que ce qui était accident devienne essence » (353-354, trad. modifiée). Le moi

19. Eckhart, Sermon allemand 83 (DW III, 447); trad. J. Ancelet-Hustache (III, 1979, 153) modifiée. Le terme « istigkeit» est un néologisme forgé par Eckhart, il peut signifier soit la forme abstraite et substantivée de "il est» (ist), soit la forme abstraite de «ceci » (ist), l’heccéité; voir Beccarisi (2003). 
comme être dans le monde, le moi désirant, connaissant et possédant n'était qu'un moi accidentel, inessentiel. Par le don divin, il est devenu essence, il est devenu le moi en tant que "moi ». Ainsi, il s'est trouvé lui-même comme moi essentiel. En un sens, il est devenu ce qu'il était déjà de toute éternité. "Lorsque la grâce eut accompli son œuvre, Paul demeura ce qu'il était " (354 modifiée). Passer de l'accidentel à l'essentiel n'est sûrement pas devenir autre que soi. Le dépassement de soi est encore une manière pour le moi de se constituer et de revenir à lui-même.

C'est alors la démarche même de se désapproprier qui est la manière dont l'homme se constitue en propre et a la certitude de soi. Eckhart n'invite pas à fuir les obligations de l'existence avec autrui, ni de la vie pratique. Il a des remarques extrêmement précises à ce sujet: «quelqu'un serait-il dans le ravissement comme jadis saint Paul, s'il apprenait qu'un infirme a besoin d'un peu de soupe qu'il pourrait lui donner, j'estime qu'il ferait bien mieux de renoncer, par charité, à son ravissement, et de servir l'indigent avec plus d'amour ${ }^{20}$ ».

Le détachement est en même temps une répétition. La désubjectivation est en réalité une subjectivation. Car au terme de ce devenir au cœur de l'absolu, l'homme est rendu à lui-même. En mettant le souci de la désappropriation au cœur de la démarche éthique, Eckhart propose une attitude fondamentale. Cette attitude ne consiste en rien de mondain, elle n'est pas «du monde », elle ne se plie pas au monde et aux différentes manières de se rapporter aux objets du monde, qui sont le désir, la possession, la connaissance. Mais elle reste une manière d'exister dans le monde. Car l'idéal d'Eckhart reste celui, très concret, de la vie pauvre, pratiquée par les dominicains et les moniales dont il est le pasteur. En les orientant vers les béatitudes de l'Évangile, avec leur signification paradoxale, Eckhart les reconduit à la béatitude par excellence. Il leur propose une attitude éthique et s'inscrit donc tout à fait rigoureusement dans la postérité d'Aristote. Simplement, l'attitude éthique en question n'aboutit pas au bonheur du sage, maître de lui-même et de ses actions, que décrit Aristote au livre X de l'Éthique à Nicomaque. Elle réside dans l'intrusion de la béatitude céleste dès ici-bas. Il nous est évidemment impossible, en tant qu'historien, de dire si une telle expérience a pu être accomplie par Eckhart ou par son auditoire. Mais il est certain que pour Eckhart lui-même, le cœur de la mystique n'est pas dans une expérience, mais dans une manière de vivre, dans un discours et dans une action conforme à ce discours. Ainsi, l'exhortation de ce

20. Entretiens spirituels, $\mathbb{1 0}$ (trad. Libera 1993, 92). 
Sermon réinstalle une polarité qui réoriente toutes les actions de l'homme consacré à l'existence croyante. Elle réinstitue l'homme selon une autre logique, selon un autre sens de la subjectivité.

Car il reste un point que nous avons négligé, en considérant simplement ce Sermon comme un ensemble d'énoncés abstraits: sa situation d'énonciation. Or, les travaux savants de Joachim Theisen (1990) nous permettent de dater ce sermon. Nous apprenons ainsi qu'il a été prononcé à l'occasion de la fête de la Toussaint: il s'insère dans le contexte liturgique de la fête de la sainteté. Eckhart signale de surcroît que ce verset n'est lui-même pas un énoncé abstrait, mais qu'il est en quelque sorte la révélation de la béatitude elle-même par elle-même. Eckhart indique en effet: "La Béatitude pleine de sagesse ouvrit la bouche et dit: Bienheureux, etc. » La béatitude n'est pas seulement un concept éthique, ou une promesse évangélique, c'est une personne, celle du Christ. Même si l'ensemble du Sermon se limite à commenter cet énoncé, nous ne pouvons oublier le double contexte d'énonciation où il s'inscrit : d'abord celui du Christ, qui révèle lui-même ce qu'il est et comment l'atteindre; puis, à distance, celui du pasteur et du théologien, qui enseigne à ses auditeurs comment, dans leur pratique quotidienne, rejoindre cette vérité transcendante et même divine, la béatitude, bref, comment imiter le Christ, comment devenir soi-même un de ces saints dont on célèbre ce jour-là la fête.

La nouvelle subjectivité à laquelle Eckhart invite ses auditeurs, même si elle est structurée par des énoncés spéculatifs extrêmement ardus, se confond tout simplement avec la sainteté. La vérité n'est pas simplement le contenu du discours énoncé par Eckhart; elle n'est pas simplement celui qui énonce ce discours (le Christ); elle est encore ce que doit devenir celui qui le comprend. Car «tant que l'homme n'est pas semblable à cette Vérité, il ne peut comprendre ce discours» (355). Devenir pauvre, c'est devenir la vérité même que décrit notre discours; et devenir ce que nous signifions, c'est devenir bienheureux. Le discours a une fonction pragmatique, du moins sur ceux qui sont assez bien disposés pour l'entendre: nous devenons ce qu'il signifie. Il nous faut comprendre la pauvreté, non comme un comportement extérieur, mais comme une disposition intérieure. Ici, comprendre et agir sur soi-même forment un cercle herméneutique. Car si nous l'intériorisons comme il convient, le discours de la béatitude peut nous rendre bienheureux. Et inversement, devenir bienheureux, c'est devenir semblable à la vérité: celle qu'énonce le sermon, mais aussi celle qui se confond avec la béatitude divine. 


\section{Références}

Aristote (2005), De l'Âme, III, trad. P. Thillet, Paris, Gallimard. (1837), Éthique à Nicomaque, Berlin, Bekker.

Augustin, Sur la Première Épître de saint Jean, Paris, Migne (Patrologie Latine [cité PL] 35).

Balmary, M. (2002), "J'ai dit: Vous êtes des dieux ", dans C. Michon, dir., Le christianisme. Héritage et destin, Paris, Libraire Générale Française, p. 393-414.

BeCCARISI, A. (2003), "Philosophische Neologismen zwischen Latein und Volkssprache: "istic" und "isticheit" bei Meister Eckhart ", Recherches de Théologie et Philosophie Médiévales, 70, p. 329-358.

Bermon, E. (2001), Le Cogito dans la pensée de saint Augustin, Paris, Vrin. BoulnoIs, O. (1999), Être et représentation, Paris, PUF, p. 151-222.

- (2008), Au-delà de l'image, Une archéologie du visuel au Moyen $\hat{A} g e, V^{e}-X V I^{e}$ siècle, Paris, Seuil (Des Travaux).

Casteigt, J. (2006), Connaissance et vérité chez Maître Eckhart. Seul le juste connaît la justice, Paris, Vrin (Études de philosophie médiévale).

ECKHART (1958 sq.), Deutsche Werke, éd. J. Quint, Stuttgart, Kohlhammer [cité DW].

(1993), Traités et sermons, trad. A. de Libera, Paris, Flammarion. (1979), Sermons, 3 vol. trad. J. Ancelet-Hustache, Paris, Seuil. (1956 sq.), Lateinische Werke, III, trad. A. de Libera, E.-H. Wéber et E. zum Brunn, Stuttgart, Kohlhammer [cité LW].

(1989), L'Euvre latine de Maître Eckhart, t. 6, Le Commentaire de l'Évangile selon Jean, Cerf, Paris.

(1984), Quaestiones parisienses, éd. et trad. Z. Kaluza et al., Maître Eckhart à Paris. Une critique médiévale de l'ontothéologie, Paris, PUF (Bibliothèque de l'École des Hautes Études, Sciences religieuses, 86).

KöBeLE, S. (1998), "Zu Predigt 16b: Quasi vas auri solidum ", Lectura Eckhardi, Predigten Meister Eckharts von Fachgelehrten gelesen und gedeutet, Stuttgart/Berlin/Cologne, G. Steer/L. Sturlese, p. 43-74.

Nazianze, G. de (1974), Lettres théologiques, éd. et trad. P. Gallay, Paris, Cerf (Sources chrétiennes, 208). 
TheISEN, J. (1990), Predigt und Gottesdienst. Liturgischen Strukturen in den Predigten Meister Eckharts, Frankfurt/New York/Paris, Peter Lang.

WaCKernagel, W. (1991), Ymagine Denudari. Éthique de l'image et Métaphysique de l'abstraction chez Maître Eckhart, Paris, Vrin.

\section{Résumé}

Le Sermon 52 d'Eckhart se situe au croisement de l'aristotélisme et de l'augustinisme: l'homme accède à la béatitude par la contemplation, il a pour modèle la divinisation du chrétien. Pour Eckhart, seul le détachement permet à l'homme d'y accéder, par un triple renoncement : à la concupiscence de la chair, à la convoitise du savoir et au désir de dominer. Dans ce détachement, devient-il un ça, un intellect abstrait, impersonnel ? Cesse-t-il d'être un moi ? Apparemment, puisque Dieu seul peut dire "moi », selon Eckhart. Mais en réalité, pas du tout, car le sermon a été prononcé pour la fête de tous les saints, unis à Dieu sur le modèle du Fils avec son Père: une seule essence, mais deux personnes.

\section{Abstract}

Eckhart's Sermon 52 is located at the intersection of Aristotelianism and Augustinism: man reaches beatitude through contemplation; his model is the deification of the Christian. For Eckhart, only detachment allows man to reach this beatitude, through a triple renunciation: of the lust for flesh, the craving for knowledge, and the desire to dominate. Does such a detachment transform him into an "it», an abstract, impersonal intellect? Does he cease to be a self? Apparently so, since God alone can say "I", according to Eckhart. But in fact, not at all, because the sermon was delivered on All Saints Day, the celebration of those united to God as the Son to the Father: a single essence, but two persons. 